PROCEEDINGS OF THE

AMERICAN MATHEMATICAL SOCIETY

Volume 134, Number 6, Pages 1809-1818

S 0002-9939(06)08433-4

Article electronically published on January 4, 2006

\title{
HAUSDORFF ULTRAFILTERS
}

\author{
MAURO DI NASSO AND MARCO FORTI
}

(Communicated by Alan Dow)

\begin{abstract}
We give the name Hausdorff to those ultrafilters that provide ultrapowers whose natural topology ( $S$-topology) is Hausdorff, e.g. selective ultrafilters are Hausdorff. Here we give necessary and sufficient conditions for product ultrafilters to be Hausdorff. Moreover we show that no regular ultrafilter over the "small" uncountable cardinal $\mathfrak{u}$ can be Hausdorff. $(\mathfrak{u}$ is the least size of an ultrafilter basis on $\omega$.) We focus on countably incomplete ultrafilters, but our main results also hold for $\kappa$-complete ultrafilters.
\end{abstract}

\section{INTRODUCTION}

Let $\mathcal{U}$ be an ultrafilter over the set $I$. Given any set $X$, the corresponding ultrapower $X^{I} / \mathcal{U}$ can be given a natural topology by taking the family of all subsets of the form $A^{I} / \mathcal{U}$ for $A \subseteq X$ as a (cl)open basis. (This topology is named $S$-topology (standard topology) in nonstandard analysis.) It turns out that the resulting topological space is Hausdorff if and only if the ultrafilter $\mathcal{U}$ satisfies a simple algebraic property, namely:

$$
\text { for all } f, g: I \rightarrow I \quad(f(\mathcal{U})=g(\mathcal{U}) \Longrightarrow f \equiv \mathcal{U} g) ! 1
$$

To the authors' knowledge, this property was first isolated for ultrafilters over $\omega$ in 8 , under the name "property $(C)$ ". There it is proved that $(C)$ holds for all 3-arrow ultrafilters, as introduced in [2]. In particular, weakly Ramsey ultrafilters, and also products of pairs of nonisomorphic selective ultrafilters, have property $(C)$. Uniform Hausdorff ultrafilters over $I$ are called separating in [13, where it is shown that they cannot be regular if $|I| \geq 2^{\aleph_{0}}$. Several large cardinal consequences are then derived from the existence of separating ultrafilters on uncountable sets. A corresponding property $S(\mathcal{I})$ for (possibly nonmaximal) ideals was studied in [16]. Focusing on uncountable cardinals $\kappa$, a combinatorial condition is given there in order to let $S(\mathcal{I})$ fail for suitable $\kappa$-complete ideals.

The Hausdorff ultrafilters came to the authors' attention in their attempts 3 , 9] to isolate a few topological properties that could characterize the nonstandard models. It is a well-known fact in nonstandard analysis that every nonstandard model $X^{*}$ can be canonically mapped into the Stone-Čech compactification $\beta X$ of the discrete space $X$ (see e.g. [17]). Starting from this fact, it is proved that

Received by the editors November 24, 2003 and, in revised form, May 12, 2004.

2000 Mathematics Subject Classification. Primary 03E05, 03H05, 54D80.

This work was partially supported by the MIUR PRIN Grant "Metodi logici nello studio di strutture geometriche, topologiche e insiemistiche", Italy.

${ }^{1}$ See Section 1 for definitions and notation. 
a nonstandard model $X^{*}$ is Hausdorff (when equipped with the $S$-topology) if and only if it is isomorphic to an invariant (i.e. Rudin-Keisler downward closed) subspace of $\beta X$ where all points (ultrafilters) satisfy the property $(\mathrm{H})$. The name Hausdorff ultrafilters was first used in [9, to stress the "topological" significance of that special class of ultrafilters. Also from a model-theoretic point of view, Hausdorff ultrafilters could be of interest. In fact their existence is equivalent to that of nonstandard extensions of the complete structure on the natural numbers where different elements have different types.

The consistency strength of Hausdorff ultrafilters seems to be an interesting problem. The countable case remained unsettled until very recently 2 On the one hand, it is easily seen that all selective ultrafilters are Hausdorff, but the two notions are different. For instance, under the Continuum Hypothesis CH (or Martin's Axiom MA), there are $2^{\mathfrak{c}}$ nonisomorphic selective ultrafilters over $\omega$. Hence, by Corollary 1.8 below, one can take their products and the many products of Hausdorff ultrafilters that are neither $P$ - nor $Q$-points. Moreover, under MA, a suitable inductive construction produces $P$-points that are not Hausdorff. However the most interesting question as to whether the existence of Hausdorff ultrafilters is provable in ZFC alone has been solved in the negative by T. Bartoszynski and S. Shelah [1, when this paper was almost completed.

Here we prove a general criterion for a product ultrafilter to be Hausdorff. In particular, under a natural incompatibility condition, Hausdorff ultrafilters are stable under products by Hausdorff $P$-points. As first pointed out in 13, Hausdorff ultrafilters on uncountable sets are highly problematic, connected as they are to irregular ultrafilters. Here we show that Hausdorff uniform ultrafilters on the small uncountable cardinal $\mathfrak{u}$ cannot be regular. ( $\mathfrak{u}$ is the least size of an ultrafilter basis on $\omega$.) However, regular uniform Hausdorff ultrafilters on $\omega_{1}$ can be constructed under Martin's axiom plus $2^{\aleph_{0}}=\aleph_{2}$. The consistency of regular uniform Hausdorff ultrafilters on cardinals greater than $\omega_{1}$ is an open problem.

The paper is organized as follows. In Section 1, we characterize those products of ultrafilters that are Hausdorff. In Section 2 we give an upper bound for the size of sets admitting regular uniform Hausdorff ultrafilters. In the final Section 3, we face the problem of delimiting the consistency strength of Hausdorff ultrafilters. We also briefly discuss $\kappa$-complete ultrafilters over an uncountable measurable cardinal $\kappa$. We conclude with a list of open questions and suggestions for further research.

\section{Products of Hausdorff ultrafilters}

While fixing notation, we recall some basic definitions and facts. Throughout this paper, $\mathcal{U}$ and $\mathcal{V}$ will denote ultrafilters on $I$ and $J$, respectively. We are mainly interested in the countable case, but all results of this section are independent of the sizes of the sets $I$ and $J$.

The additivity of a nonprincipal ultrafilter $\mathcal{U}$ is the least amount of sets outside $\mathcal{U}$ having union in $\mathcal{U} . \mathcal{U}$ is $\kappa$-complete if its additivity is at least $\kappa$, i.e. $\mathcal{U}$ is (nonprincipal and) closed under intersections of size less than $\kappa$. It is well known that

\footnotetext{
${ }^{2}$ The question has been posted by the authors to the "Logic café" of Logic Colloquium '03, Helsinki.
} 
the additivity of any ultrafilter is a measurable cardinal 3 Ultrafilters of additivity $\omega$ are usually called countably incomplete.

Two functions $f$ and $g$ with domain $I$ are equivalent modulo $\mathcal{U}$ (or $\mathcal{U}$-equivalent) iff $\{i \in I \mid f(i)=g(i)\} \in \mathcal{U}$. In this case we write $f \equiv \mathcal{U} g$. If $f: I \rightarrow J$, the family $f(\mathcal{U})=\left\{B \subseteq J \mid f^{-1}(B) \in \mathcal{U}\right\}$ is an ultrafilter on $J$ The Rudin-Keisler preordering on ultrafilters is defined by setting $\mathcal{V} \leq_{R K} \mathcal{U}$ whenever $\mathcal{V}=f(\mathcal{U})$ for some $f$. In this case we say that $\mathcal{V}$ is $R K$-below $\mathcal{U}$ (or $\mathcal{U}$ is $R K$-above $\mathcal{V}$ ). It is a basic fact in the theory of ultrafilters that $f(\mathcal{U})=\mathcal{U} \Leftrightarrow f \equiv_{\mathcal{U}} i d$, where $i d$ denotes the identity map. As a consequence, both $\mathcal{U} \leq_{R K} \mathcal{V}$ and $\mathcal{V} \leq_{R K} \mathcal{U}$ hold if and only if $f(\mathcal{U})=\mathcal{V}$ for some $f: I \rightarrow J$ which is 1-1 when restricted to a suitable $U \in \mathcal{U}$. In this case $\mathcal{U}$ and $\mathcal{V}$ are isomorphic, and we write $\mathcal{U} \cong$ $\mathcal{V}$. Thus $\leq_{R K}$ becomes a partial order on the equivalence classes of isomorphic ultrafilters. We say that $\mathcal{U}, \mathcal{V}$ are incomparable if $\mathcal{U} \mathbb{Z}_{R K} \mathcal{V}$ and $\mathcal{V} \mathbb{Z}_{R K} \mathcal{U}$; they are incompatible if there is no nonprincipal ultrafilter $\mathcal{W} \leq_{R K} \mathcal{U}, \mathcal{V}$. A nonprincipal ultrafilter $\mathcal{U}$ is selective (or Ramsey) if every $f: I \rightarrow I$ is $\mathcal{U}$-equivalent either to a constant or to a 1-1 function. Selective ultrafilters are exactly the minimal elements of the $R K$-preordering, i.e. those lying immediately above the principal ultrafilters. An ultrafilter is uniform if all its elements have the same cardinality. Every nonprincipal ultrafilter is isomorphic to a uniform ultrafilter over $\kappa$, where $\kappa$ is the least size of a set in $\mathcal{U}$. However in the following we shall not restrict ourselves to uniform ultrafilters.

The product filter $\mathcal{U} \times \mathcal{V}$ is the filter on $I \times J$ generated by all products $U \times V$ where $U \in \mathcal{U}$ and $V \in \mathcal{V}$. Notice that, in general, $\mathcal{U} \times \mathcal{V}$ is not ultra. The product ultrafilter $\mathcal{U} \otimes \mathcal{V}$ (sometimes called tensor product) is defined by setting

$$
X \in \mathcal{U} \otimes \mathcal{V} \Longleftrightarrow\{i \in I \mid\{j \in J \mid(i, j) \in X\} \in \mathcal{V}\} \in \mathcal{U}
$$

Let $\left\{\mathcal{V}_{i} \mid i \in I\right\}$ be a family of ultrafilters on $J$, and $\mathcal{U}$ an ultrafilter on $I$. The $\mathcal{U}$-limit $\lim _{\mathcal{U}} \mathcal{V}_{i}$ of the family is the ultrafilter on $J$ such that

$$
X \in \lim _{\mathcal{U}} \mathcal{V}_{i} \Longleftrightarrow\left\{i \in I \mid X \in \mathcal{V}_{i}\right\} \in \mathcal{U}
$$

Clearly, $\lim _{\mathcal{U}} \mathcal{V}_{i}=\lim _{\mathcal{U}} \mathcal{W}_{i}$ whenever $\left\{i \in I \mid \mathcal{V}_{i}=\mathcal{W}_{i}\right\} \in \mathcal{U}$

If not explicitly mentioned otherwise, we refer to [10] for all set-theoretic notions and facts used in this paper, and to [7] for definitions and facts concerning ultrapowers, ultrafilters, and nonstandard models.

Let us repeat our basic definition of the Hausdorff ultrafilter:

Definition 1.1. An ultrafilter $\mathcal{U}$ is Hausdorff if it satisfies the condition:

$$
\text { for all } f, g: I \rightarrow I \quad(f(\mathcal{U})=g(\mathcal{U}) \Longrightarrow f \equiv \mathcal{U} g) \text {. }
$$

Note that the above condition $(\mathrm{H})$ is automatically satisfied whenever any of the functions $f$ and $g$ is $1-1$ or constant when restricted to some set $A \in \mathcal{U}$. In particular, all selective ultrafilters are Hausdorff. It is easily seen that Hausdorff ultrafilters are downward closed with respect to the Rudin-Keisler preordering.

We begin with a simple characterization of Hausdorff ultrafilters.

\footnotetext{
${ }^{3} \mathrm{~A}$ cardinal $\kappa$ is measurable if there is a nonprincipal $\kappa$-complete ultrafilter on $\kappa$. (Note that we include $\omega$ among the measurable cardinals.)

${ }^{4}$ The notation $f_{*}(\mathcal{U})$ is frequently used in the literature. We adhere to the simpler notation $f(\mathcal{U})$ since no ambiguity is likely to arise.
} 
Lemma 1.2. The ultrafilter $\mathcal{U}$ is Hausdorff if and only if whenever an ultrafilter $\mathcal{W} \leq_{R K} \mathcal{U}$ includes the square filter $\mathcal{V} \times \mathcal{V}$ of some nonprincipal $\mathcal{V}$, then $\mathcal{W}$ also includes the diagonal $\Delta=\{(j, j) \mid j \in J\}$.

Proof. Assume that $f: I \rightarrow J \times J$ is a function such that $\mathcal{V} \times \mathcal{V} \subseteq f(\mathcal{U})=\mathcal{W}$. Let $\pi_{1}, \pi_{2}: J \times J \rightarrow J$ be the canonical projections. Then $\pi_{1}(\mathcal{W})=\pi_{2}(\mathcal{W})=\mathcal{V}$, while $\pi_{1} \equiv \mathcal{W} \pi_{2}$ if and only if $\mathcal{W}$ includes the diagonal $\Delta \subseteq J \times J$. Since Hausdorff ultrafilters are Rudin-Keisler downward closed, we obtain the direct implication.

Conversely, let $f, g: I \rightarrow J$ be such that $f(\mathcal{U})=g(\mathcal{U})=\mathcal{V}$ is nonprincipal. Define $h: I \rightarrow J \times J$ by $h(i)=(f(i), g(i))$. Then clearly $\mathcal{W}=h(\mathcal{U}) \supseteq \mathcal{V} \times \mathcal{V}$, and so $\mathcal{W}$ includes the diagonal. But then $f$ and $g$ are equivalent modulo $\mathcal{U}$, since they agree on $h^{-1}(\Delta) \in \mathcal{U}$. Therefore $\mathcal{U}$ is Hausdorff.

In particular all power ultrafilters $\mathcal{U}^{\otimes k}=\mathcal{U} \otimes \cdots \otimes \mathcal{U}(k$ times $)$ are non-Hausdorff, as already remarked in [8]. More generally

Corollary 1.3. If the product $\mathcal{U} \otimes \mathcal{V}$ is Hausdorff, then the ultrafilters $\mathcal{U}$ and $\mathcal{V}$ are Hausdorff and incompatible.

Proof. If $\mathcal{W}=\mathcal{U} \otimes \mathcal{V}$ is Hausdorff, then both $\mathcal{U}$ and $\mathcal{V}$ are $\leq_{R K} \mathcal{W}$, hence Hausdorff. If $f: I \rightarrow K, g: J \rightarrow K$ are such that $f(\mathcal{U})=g(\mathcal{V})=\mathcal{D}$, define $h: I \times J \rightarrow K \times K$ by $h(i, j)=(f(i), g(j))$. Then the ultrafilter $h(\mathcal{W})$ includes $\mathcal{D} \times \mathcal{D}$, hence also the diagonal, according to Lemma 1.2, Now

$$
h^{-1}(\Delta)=\{(i, j) \mid f(i)=g(j)\} \in \mathcal{U} \otimes \mathcal{V} \Longleftrightarrow\{i \mid\{j \mid f(i)=g(j)\} \in \mathcal{V}\} \in \mathcal{U} .
$$

But this implies that both $f$ and $g$ are constant, modulo $\mathcal{U}$ and $\mathcal{V}$ respectively, and so $\mathcal{D}$ is principal. This proves that $\mathcal{U}$ and $\mathcal{V}$ are incompatible.

Our criterion to test whether a product of Hausdorff ultrafilters is itself Hausdorff is obtained by adding a further condition, namely:

Theorem 1.4. The product $\mathcal{U} \otimes \mathcal{V}$ is Hausdorff if and only if the following conditions are satisfied:

(i) $\mathcal{U}$ and $\mathcal{V}$ are Hausdorff and incompatible;

(ii) for $i \in I$, let $\mathcal{V}_{i}^{\prime} \neq \mathcal{V}_{i}^{\prime \prime}$ be (possibly principal) ultrafilters on $J$ that are $\leq_{R K} \mathcal{V}$. Then $\lim _{\mathcal{U}} \mathcal{V}_{i}^{\prime} \neq \lim _{\mathcal{U}} \mathcal{V}_{i}^{\prime \prime}$.

Proof. Assume first that the product $\mathcal{U} \otimes \mathcal{V}$ is Hausdorff. Condition (i) holds by Corollary 1.3. Proceeding by contradiction, let the ultrafilters $\mathcal{V}_{i}^{\prime}=f_{i}(\mathcal{V})$, $\mathcal{V}_{i}^{\prime \prime}=g_{i}(\mathcal{V})$ witness that condition (ii) fails. Put $f(i, j)=f_{i}(j)$ and $g(i, j)=g_{i}(j)$. A straightforward application of the definitions proves the equalities

$$
f(\mathcal{U} \otimes \mathcal{V})=\lim _{\mathcal{U}} \mathcal{V}_{i}^{\prime}=\lim _{\mathcal{U}} \mathcal{V}_{i}^{\prime \prime}=g(\mathcal{U} \otimes \mathcal{V})
$$

Now $f \equiv \mathcal{U} \otimes \mathcal{V} g$ if and only if $\left\{i \mid f_{i} \equiv \mathcal{V} g_{i}\right\} \in \mathcal{U}$. But the latter condition is not realized, because $f_{i}(\mathcal{V})=\mathcal{V}_{i}^{\prime} \neq \mathcal{V}_{i}^{\prime \prime}=g_{i}(\mathcal{V})$, and so $f_{i} \not \equiv \mathcal{V} g_{i}$, for all $i$. Thus we obtain the contradiction that $\mathcal{U} \otimes \mathcal{V}$ is not Hausdorff.

Vice versa, let $\mathcal{U}$ and $\mathcal{V}$ satisfy conditions (i) and (ii). Given $f, g: I \times J \rightarrow K$, define the functions $f_{i}, g_{i}: J \rightarrow K$ by $f_{i}(j)=f(i, j)$ and $g_{i}(j)=g(i, j)$. Since $\mathcal{V}$ is Hausdorff, $\left\{i \mid f_{i}(\mathcal{V}) \neq g_{i}(\mathcal{V})\right\}=\left\{i \mid f_{i} \not \equiv \mathcal{V} g_{i}\right\} \in \mathcal{U}$ if and only if $f \not \equiv \mathcal{U} \otimes \mathcal{V} g$. In this case, take two sequences of ultrafilters $\mathcal{V}_{i}^{\prime}, \mathcal{V}_{i}^{\prime \prime}$ such that $\mathcal{V}_{i}^{\prime} \neq \mathcal{V}_{i}^{\prime \prime}$ for all $i$, and $\mathcal{V}_{i}^{\prime}=f_{i}(\mathcal{V}), \mathcal{V}_{i}^{\prime \prime}=g_{i}(\mathcal{V})$ whenever $f_{i} \not \equiv \mathcal{V} g_{i}$. By condition (ii), we conclude that $f(\mathcal{U} \otimes \mathcal{V})=\lim _{\mathcal{U}} f_{i}(\mathcal{V})=\lim _{\mathcal{U}} \mathcal{V}_{i}^{\prime} \neq \lim _{\mathcal{U}} \mathcal{V}_{i}^{\prime \prime}=\lim _{\mathcal{U}} g_{i}(\mathcal{V})=g(\mathcal{U} \otimes \mathcal{V})$. 
Note also that principal ultrafilters are allowed in condition (ii). As a consequence, that condition already implies that the ultrafilters $\mathcal{U}$ and $\mathcal{V}$ are incompatible. In fact, if $f(\mathcal{U})=g(\mathcal{V})$ is nonprincipal, let $\mathcal{V}_{i}^{\prime}$ be the principal ultrafilter generated by $\{f(i)\}$ and let $\mathcal{V}_{i}^{\prime \prime}=g(\mathcal{V})$ for all $i \in I$. Then clearly $\lim _{\mathcal{U}} \mathcal{V}_{i}^{\prime}=\lim _{\mathcal{U}} \mathcal{V}_{i}^{\prime \prime}$, contradicting condition (ii). In order to simplify the above criterion, the following notions are useful.

Definition 1.5. An indexed family $\left\{\mathcal{V}_{i} \mid i \in I\right\}$ of ultrafilters over $J$ is discrete if there exists a family $\left\{A_{i} \mid i \in I\right\}$ of subsets of $J$ such that $A_{i} \in \mathcal{V}_{i}$ and $A_{i} \cap A_{j}=\emptyset$ whenever $\mathcal{V}_{i} \neq \mathcal{V}_{j}$. (Note that we do not assume that $i \neq j$ implies $\mathcal{V}_{i} \neq \mathcal{V}_{j}$.) The family $\left\{\mathcal{V}_{i} \mid i \in I\right\}$ is $\mathcal{U}$-compatible if there exists a nonprincipal ultrafilter $\mathcal{W}$ such that $\left\{i \in I \mid \mathcal{W} \leq_{R K} \mathcal{V}_{i}\right\} \in \mathcal{U}$.

Theorem 1.6. Let $\mathcal{U}, \mathcal{V}$ be incompatible Hausdorff ultrafilters, and let $\kappa$ be the least size of a set in $\mathcal{U}$. Then the product $\mathcal{U} \otimes \mathcal{V}$ is Hausdorff if the following conditions are satisfied for every family $\left\{\mathcal{V}_{i} \mid i \in I\right\}$ of ultrafilters $\leq \mathcal{V}$ :

(a) every subfamily of size $\kappa$ is discrete;

(b) if $\lim _{\mathcal{U}} \mathcal{V}_{i} \leq_{R K} \mathcal{U}$, then the family $\left\{\mathcal{V}_{i} \mid i \in I\right\}$ is $\mathcal{U}$-compatible.

Proof. Let $\left\{\mathcal{V}_{i}^{\prime} \mid i \in I\right\}$ and $\left\{\mathcal{V}_{i}^{\prime \prime} \mid i \in I\right\}$ be families of ultrafilters satisfying the hypotheses of condition (ii) of Theorem 1.4. Put $A^{\prime}=\left\{i \in I \mid \mathcal{V}_{i}^{\prime}\right.$ principal $\}$ and $A^{\prime \prime}=\left\{i \in I \mid \mathcal{V}_{i}^{\prime \prime}\right.$ principal $\}$. We show that (a) and (b) together imply (ii). We distinguish three cases.

Case 1. $A^{\prime} \in \mathcal{U}$ and $A^{\prime \prime} \in \mathcal{U}$. Pick functions $f, g: I \rightarrow J$ such that $\{f(i)\} \in \mathcal{V}_{i}^{\prime}$ and $\{g(i)\} \in \mathcal{V}_{i}^{\prime \prime}$ for all $i \in A^{\prime} \cap A^{\prime \prime}$. As $f \not \mathcal{U}_{\mathcal{U}} g$ and $\mathcal{U}$ is Hausdorff, we have

$$
\lim _{\mathcal{U}} \mathcal{V}_{i}^{\prime}=f(\mathcal{U}) \neq g(\mathcal{U})=\lim _{\mathcal{U}} \mathcal{V}_{i}^{\prime \prime}
$$

So condition (ii) of Theorem 1.4 is satisfied.

Case 2. $A^{\prime} \notin \mathcal{U}$ and $A^{\prime \prime} \notin \mathcal{U}$. Assume w.l.o.g. that all $\mathcal{V}_{i}^{\prime}, \mathcal{V}_{i}^{\prime \prime}$ are nonprincipal. Enumerate

$$
\left\{\mathcal{V}_{i}^{\prime} \mid i \in I\right\} \cup\left\{\mathcal{V}_{i}^{\prime \prime} \mid i \in I\right\}=\left\{\mathcal{W}_{h} \mid h \in H\right\}
$$

in such a way that $\mathcal{W}_{h} \neq \mathcal{W}_{k}$ for $h \neq k$. Let $\sigma, \tau: I \rightarrow H$ be the functions such that $\mathcal{V}_{i}^{\prime}=\mathcal{W}_{\sigma(i)}$ and $\mathcal{V}_{i}^{\prime \prime}=\mathcal{W}_{\tau(i)}$. Note that $\lim _{\mathcal{U}} \mathcal{V}_{i}^{\prime}=\lim _{\sigma(\mathcal{U})} \mathcal{W}_{h}$ and $\lim _{\mathcal{U}} \mathcal{V}_{i}^{\prime \prime}=\lim _{\tau(\mathcal{U})} \mathcal{W}_{h}$. Since $\sigma \not \neq_{\mathcal{U}} \tau$ and $\mathcal{U}$ is Hausdorff, it follows that $\sigma(\mathcal{U}) \neq$ $\tau(\mathcal{U})$, so we can find a set $A \in \mathcal{U}$ of size $\kappa$ such that $\sigma(A) \cap \tau(A)=\emptyset$. The family $\left\{\mathcal{W}_{h} \mid h \in \sigma(A) \cup \tau(A)\right\}$ is discrete, by condition (a). Choose a family $\left\{A_{h} \mid h \in \sigma(A) \cup \tau(A)\right\}$ of subsets of $J$ with the property that $A_{h} \in \mathcal{W}_{h}$, and $A_{h} \cap A_{k}=\emptyset$ for $h \neq k$. Put $X=\bigcup_{h \in \sigma(A)} A_{h}$ and $Y=\bigcup_{h \in \tau(A)} A_{h}$. Then $X \in \lim _{\sigma(\mathcal{U})} \mathcal{W}_{h}, Y \in \lim _{\tau(\mathcal{U})} \mathcal{W}_{h}$, and $X \cap Y=\emptyset$. Hence the limits are different, and condition (ii) of Theorem 1.4 is fulfilled also in this case.

Case 3. $A^{\prime} \notin \mathcal{U}$ and $A^{\prime \prime} \in \mathcal{U}$ (the case $A^{\prime} \in \mathcal{U}$ and $A^{\prime \prime} \notin \mathcal{U}$ is symmetric). Assume w.l.o.g. that all $\mathcal{V}_{i}^{\prime}$ are nonprincipal. As in Case 1, pick a function $g: I \rightarrow J$ such that $\lim _{\mathcal{U}} \mathcal{V}_{i}^{\prime \prime}=g(\mathcal{U})$. If $\lim _{\mathcal{U}} \mathcal{V}_{i}^{\prime}=\lim _{\mathcal{U}} \mathcal{V}_{i}^{\prime \prime} \leq_{R K} \mathcal{U}$, then pick a nonprincipal $\mathcal{W}$ (on $K$, say) witnessing that the condition (b) is fulfilled. Let $A \in \mathcal{U}$ be a set of size $\kappa$ such that $\mathcal{W} \leq_{R K} \mathcal{V}_{i}^{\prime}$ for all $i \in A$. Choose functions $f_{i}: J \rightarrow K$ such that $f_{i}\left(\mathcal{V}_{i}^{\prime}\right)=\mathcal{W}$ and subsets $B_{i} \subseteq J$ witnessing that the family $\left\{\mathcal{V}_{i}^{\prime} \mid i \in A\right\}$ is discrete. Pick a function $f: J \rightarrow K$ that agrees with $f_{i}$ on $B_{i}$, for every $i \in A$. Then

$$
\mathcal{W}=\lim _{\mathcal{U}} f_{i}\left(\mathcal{V}_{i}^{\prime}\right)=\lim _{\mathcal{U}} f\left(\mathcal{V}_{i}^{\prime}\right)=f\left(\lim _{\mathcal{U}} \mathcal{V}_{i}^{\prime}\right) \leq_{R K} \lim _{\mathcal{U}} \mathcal{V}_{i}^{\prime} \leq_{R K} \mathcal{U}
$$


But obviously $\mathcal{W} \leq_{R K} \mathcal{V}$, contradicting the incompatibility of $\mathcal{U}$ and $\mathcal{V}$.

Call a $\kappa$-P-point a $\kappa$-complete ultrafilter $\mathcal{U}$ on $I$ such that every function $f: I \rightarrow$ $I$ is $\mathcal{U}$-equivalent either to a constant or to a " $<\kappa$-to-1" function. (We say that $f$ is $<\kappa-t o-1$ if the fibers of $f$ are "small", i.e. $\left|f^{-1}(i)\right|<\kappa$ for all $i \in I$.) Obviously every $\kappa$-complete selective ultrafilter is a $\kappa$-P-point. In particular, $\mathcal{U}$ is a uniform selective ultrafilter on the uncountable set $I$ if and only if $\kappa=|I|$ is measurable, and $\mathcal{U}$ is isomorphic to a normal $\kappa$-ultrafilter (see e.g. 11]). When $\mathcal{V}$ is a $\kappa$-P-point, the necessary condition (i) of Thorem 1.4 becomes sufficient:

Corollary 1.7. Let $\kappa$ be the least size of a set in $\mathcal{U}$ and let $\mathcal{V}$ be a $\kappa$-P-point. Then $\mathcal{U} \otimes \mathcal{V}$ is Hausdorff if and only if $\mathcal{U}$ and $\mathcal{V}$ are Hausdorff and incompatible.

Proof. It is well known that a family of size not exceeding $\kappa$ of $\kappa$-P-points is discrete (see e.g. [1]). Moreover a $\kappa$-P-point is selective whenever its additivity exceeds $\kappa$. On the other hand a $\kappa$-P-point of additivity $\kappa$ is isomorphic to a $\kappa$-ultrafilter, i.e. a uniform $\kappa$-complete ultrafilter over $\kappa$. So, if the additivity of $\mathcal{V}$ is uncountable, there is a unique normal ultrafilter lying $R K$-below $\mathcal{V}$ (see e.g. [14]), which is, up to isomorphism, the least nonprincipal ultrafilter $\leq_{R K} \mathcal{V}$. On the other hand, if $\mathcal{V}$ is countably incomplete, and so $\kappa=\aleph_{0}$, it follows from Theorem 5 of 4 that any sequence of nonprincipal ultrafilters $\leq_{R K} \mathcal{V}$ has a nonprincipal $R K$-lower bound $\mathcal{W} \leq_{R K} \mathcal{V}$. Therefore conditions (a)-(b) of Theorem 1.6 are always met.

Similarly, if $\mathcal{U}$ is a $\kappa$-P-point, then condition (b) of Theorem[1.6 is trivial, because only constant $\kappa$-sequences can converge to $\kappa$-P-points, and so (a) alone suffices for $\mathcal{U} \otimes \mathcal{V}$ to be Hausdorff. More generally, provided sufficiently many incompatible Hausdorff $\kappa$-P-points are available, one can produce other Hausdorff ultrafilters that are (Rudin-Keisler) greater and greater. Namely, by recalling that a $P$-point lies $R K$-below a product if and only if it lies $R K$-below some of the factors (see e.g. [6]), we can prove by induction:

Corollary 1.8. Let $\left\{\mathcal{U}_{h} \mid h \in H\right\}$ be a family of pairwise incompatible Hausdorff $\kappa$-P-points over $\kappa$. Then the product $\mathcal{U}_{h_{1}} \otimes \ldots \otimes \mathcal{U}_{h_{n}}$ is Hausdorff if and only if the indices $h_{1}, \ldots, h_{n}$ are all different.

Any product of nonprincipal ultrafilters is neither a $P$ - nor a $Q$-point. Thus, whenever we have two nonisomorphic selective ultrafilters, we also have a Hausdorff ultrafilter that is neither a $P$ - nor a $Q$-point.

\section{Regular Hausdorff ultrafilters}

Recall that the ultrafilter $\mathcal{U}$ on $I$ is $(\kappa, \nu)$-regular if there exists a family $\mathcal{F} \subseteq \mathcal{U}$ of size $\nu$ such that the intersection of any subfamily of size $\kappa$ of $\mathcal{F}$ is empty. $\mathcal{U}$ is regular if it is $(\omega,|I|)$-regular, i.e. if there is a family $\left\{A_{i} \mid i \in I\right\} \subset \mathcal{U}$ such that every infinite subfamily has empty intersection. Every regular ultrafilter is countably incomplete, and when $I$ is countable the two notions are equivalent (but in general regularity is a proper strengthening of countable incompleteness).

According to [13, a uniform ultrafilter $\mathcal{U}$ on $I$ is $\lambda$-separating if, whenever the functions $f, g: I \rightarrow \lambda$ are such that $f(\mathcal{U})=g(\mathcal{U})$ is uniform, then $f \equiv \mathcal{U} g$. It is easily seen that $\mathcal{U}$ is Hausdorff if and only if it is $\lambda$-separating for all $\lambda \leq|I|$. Uniform Hausdorff ultrafilters are called separating in [13]. For cardinals $\kappa \leq \lambda$ put

$\mathfrak{u}_{\kappa \lambda}=$ the least size of a $\kappa$-subbasis of a uniform $\kappa$-complete ultrafilter on $\lambda$. 
( $\mathcal{B}$ is a $\kappa$-subbasis of the filter $\mathcal{F}$ if the intersections of less than $\kappa$ elements of $\mathcal{B}$ generate $\mathcal{F}$.) Note that the cardinal $\mathfrak{u}_{\kappa \lambda}$ may be undefined for some (all) $\lambda$, when $\kappa$ is uncountable. Clearly $\mathfrak{u}=\mathfrak{u}_{\omega \omega}$ is the least size of an ultrafilter basis on any set $I$. Since obviously $\mathfrak{u}_{\kappa \lambda} \leq 2^{\lambda}$, the following theorem generalizes and improves Theorem 2.5 of 13 :

Theorem 2.1. There are no $\left(\kappa, \mathfrak{u}_{\kappa \lambda}\right)$-regular $\lambda$-separating $\kappa$-complete ultrafilters. In particular, if $|I| \geq \mathfrak{u}$, no regular ultrafilter on I is Hausdorff.

Proof. Let $\mathcal{U}$ be a $\left(\kappa, \mathfrak{u}_{\kappa \lambda}\right)$-regular $\kappa$-complete ultrafilter on $I$. Let $\left\{A_{\alpha} \mid \alpha<\mathfrak{u}_{\kappa \lambda}\right\}$ be a family of $\mathfrak{u}_{\kappa \lambda}$-many sets in $\mathcal{U}$ such that every subfamily of size $\kappa$ has an empty intersection. For $i \in I$ put $F_{i}=\left\{\alpha<\mathfrak{u}_{\kappa \lambda} \mid i \in A_{\alpha}\right\}$. Then $F_{i}$ has size less than $\kappa$, and we can assume w.l.o.g. that it is nonempty. Let $\mathcal{B}=\left\{B_{\alpha} \mid \alpha<\mathfrak{u}_{\kappa \lambda}\right\}$ be a $\kappa$-subbasis of a uniform $\kappa$-complete ultrafilter $\mathcal{D}$ on $\lambda$. Choose functions $f, g: I \rightarrow \lambda$ such that $f(i), g(i) \in \bigcap_{\alpha \in F_{i}} B_{\alpha}$ and $f(i) \neq g(i)$ for all $i \in I$. Then clearly every $B_{\alpha} \in \mathcal{B}$ belongs both to $f(\mathcal{U})$ and $g(\mathcal{U})$. Hence $f(\mathcal{U})=g(\mathcal{U})=\mathcal{D}$, but $f \neq \equiv \mathcal{U} g$, and $\mathcal{U}$ cannot be $\lambda$-separating.

The cardinal $\mathfrak{u}$ is one of several cardinal invariants of the continuum considered in the literature (see [19, [5]). As usual for cardinals that are the sizes of distinguished subsets of $\mathcal{P}(\omega)$, all that is provable in ZFC about $\mathfrak{u}$ is that $\aleph_{1} \leq \mathfrak{u} \leq 2^{\aleph_{0}}$ (see [5]). So, under $\mathbf{C H}$, there are no $\left(\omega, \omega_{1}\right)$-regular Hausdorff ultrafilters. In fact, uniform Hausdorff ultrafilters on the continuum require very strong set theoretic hypotheses. In [13] one derives from the existence of such an ultrafilter that, under $\mathrm{CH}$, there are inner models with measurable cardinals. Even when the continuum is larger than $\aleph_{1}$, one could expect that similar results are derivable from Theorem 2.1] E.g. if $2^{\aleph_{0}}=2^{\aleph_{1}}=\aleph_{2}$ and $\mathfrak{u}=\aleph_{1}$, then an argument similar to those of [13] yields the same implication. On the other hand, in [12] it is proved that if $\diamond$ holds and there is a normal $\omega_{1}$-dense ideal over $\omega_{1} 5$ then for any nonprincipal ultrafilter $\mathcal{D}$ on $\omega$ there exists a uniform ultrafilter $\mathcal{U}$ on $\mathbb{R}$ such that $\mathcal{V}<_{R K} \mathcal{U}$ implies $\mathcal{V} \leq_{R K} \mathcal{D}$. Since $\diamond$ implies $\mathrm{CH}$, one can choose $\mathcal{D}$ to be Hausdorff, and so $\mathcal{U}$ is a uniform Hausdorff ultrafilter over $\mathbb{R}$. On the contrary, when the continuum is "large" and $\mathfrak{u}=2^{\aleph_{0}}$, the existence of Hausdorff uniform ultrafilters over "large" sets should be obtained by very weak assumtions. E.g., Martin's Axiom together with $2^{\aleph_{0}}=\aleph_{2}$ yields the existence of regular uniform Hausdorff ultrafilters over $\omega_{1} 6$

\section{Final Remarks and open Questions}

3.1. Sums of Hausdorff ultrafilters. Let $\left\{\mathcal{V}_{i} \mid i \in I\right\}$ be a family of ultrafilters on $J$. The $\mathcal{U}$-sum ultrafilter $\sum_{\mathcal{U}} \mathcal{V}_{i}$ is the ultrafilter on $I \times J$ defined as follows:

$$
X \in \sum_{\mathcal{U}} \mathcal{V}_{i} \Longleftrightarrow\left\{i \in I \mid\{j \in J \mid(i, j) \in X\} \in \mathcal{V}_{i}\right\} \in \mathcal{U}
$$

Note that when all $\mathcal{V}_{i}=\mathcal{V}$, then $\sum_{\mathcal{U}} \mathcal{V}_{i}=\mathcal{U} \otimes \mathcal{V}$

The results of Section 1 can be easily extended to ultrafilter sums. We omit the proofs, which are straightforward modifications of the corresponding proofs of Section 1 .

\footnotetext{
${ }^{5}$ The consistency of this hypothesis has been proved by Woodin by assuming $A D_{\mathbb{R}}+$ " $\Theta$ is regular".

${ }^{6}$ Personal communication by K. Hrbàček.
} 
Theorem 3.1. The sum $\sum_{\mathcal{U}} \mathcal{V}_{i}$ is Hausdorff if and only if the following conditions are satisfied:

(i) $\mathcal{U}$ is Hausdorff and $\left\{i \in I \mid \mathcal{V}_{i}\right.$ is Hausdorff $\} \in \mathcal{U}$.

(ii) Let $\mathcal{V}_{i}^{\prime} \neq \mathcal{V}_{i}^{\prime \prime}$ be (possibly principal) ultrafilters on $J$ such that $\mathcal{V}_{i}^{\prime}, \mathcal{V}_{i}^{\prime \prime} \leq_{R K} \mathcal{V}_{i}$ for all $i \in I$. Then $\lim _{\mathcal{U}} \mathcal{V}_{i}^{\prime} \neq \lim _{\mathcal{U}} \mathcal{V}_{i}^{\prime \prime}$.

Recall that a family $\left\{\mathcal{V}_{i} \mid i \in I\right\}$ of ultrafilters is $\mathcal{U}$-incompatible if it is not $\mathcal{U}$-compatible, i.e. if $\left\{i \in I \mid \mathcal{W} \leq_{R K} \mathcal{V}_{i}\right\} \in \mathcal{U}$ only holds when $\mathcal{W}$ is principal.

Theorem 3.2. Let $\left\{\mathcal{V}_{i} \mid i \in I\right\}$ be a $\mathcal{U}$-incompatible family of Hausdorff ultrafilters, and let $\kappa$ be the least size of a set in $\mathcal{U}$. Then the sum $\sum_{\mathcal{U}} \mathcal{V}_{i}$ is Hausdorff if the following conditions are fulfilled for every choice of nonprincipal ultrafilters $\mathcal{V}_{i}^{\prime} \leq_{R K} \mathcal{V}_{i}$ :

(a) Every subfamily of size $\kappa$ is discrete.

(b) $\lim _{\mathcal{U}} \mathcal{V}_{i}^{\prime} \mathbb{Z}_{R K} \mathcal{U}$

Corollary 3.3. Let $\mathcal{U}$ be a Hausdorff $\kappa$-P-point and let $\left\{\mathcal{V}_{i} \mid i \in I\right\}$ be a $\mathcal{U}$ incompatible family of Hausdorff $\kappa$-P-points. Then the sum $\sum_{\mathcal{U}} \mathcal{V}_{i}$ is Hausdorff.

Having so treated those Hausdorff ultrafilters that are Rudin-Frolik composite, the next natural step would be to investigate Hausdorff $P$-points. We cannot enter into this topic here; that we aim to consider in a separate paper.

3.2. Uncountably complete Hausdorff ultrafilters. We now deal briefly with $\kappa$-ultrafilters, i.e. $\kappa$-complete ultrafilters on sets of measurable size $\kappa>\omega$. Ultrapowers modulo such ultrafilters preserve well-foundedness, and this fact has several important consequences. In particular the natural ordering on the ultrapower $\kappa^{\kappa} / \mathcal{U}$ is a well ordering, and this is the main tool used in the investigation of $\kappa$-complete ultrafilters (see [15, 14, 11]). Moreover also the Rudin-Keisler preordering on $\kappa$ ultrafilters is well founded, and so every $R K$-descending chain is finite, and ends with a selective (normal) one. If $\mathcal{U}$ is a $\kappa$-P-point, then there is exactly one normal $\kappa$-ultrafilter $\mathcal{D} \leq_{R K} \mathcal{U}$, namely $\mathcal{D}=f(\mathcal{U})$, where $f$ is the least nonconstant function in $\kappa^{\kappa} / \mathcal{U}$. In particular the existence of $\omega_{1}$-complete Hausdorff ultrafilters is equivalent to that of uncountable measurable cardinals. Moreover, if $\mathcal{U}$ is a normal $\kappa$-ultrafilter, then in $L[\mathcal{U}]$ any $\kappa$-ultrafilter is a power of $\mathcal{U}$ (see [15]), and so $\mathcal{U}$ is simultaneously the unique $\kappa$-P-point, the unique $\kappa$ - $Q$-point, and the unique Hausdorff $\kappa$-ultrafilter.

The above results on products and sums also hold for $\kappa$-ultrafilters. Besides, due to the mentioned properties, some of them admit simplified formulations. E.g.

Corollary 3.4. Let $\mathcal{U}$ be a Hausdorff $\kappa$-ultrafilter and let $\mathcal{V}$ be a Hausdorff $\kappa$-Ppoint $R K$-above the normal $\kappa$-ultrafilter $\mathcal{D}$. Then $\mathcal{U} \otimes \mathcal{V}$ is Hausdorff if and only if $\mathcal{D} \mathbb{Z}_{R K} \mathcal{U}$.

3.3. The consistency strength of Hausdorff ultrafilters. It is well known that, under $\mathrm{CH}$ or $\mathrm{MA}$, there are $2^{\mathfrak{c}}$ nonisomorphic selective ultrafilters over $\omega$. So, under any of these hypotheses, Corollary 1.8 provides equally many Hausdorff ultrafilters that are not $P$-points. Another interesting consequence is that there are strictly $R K$-increasing sequences of Hausdorff ultrafilters (which cannot be 3 arrow; see 2]). Taking direct limits of such sequences, one produces invariant analytic coherent nonprincipal subspaces of $\beta \omega$ in the sense of [9], thus answering a question posed there. Moreover, under the same hypotheses, one can inductively 
define both Hausdorff and non-Hausdorff nonselective $P$-points. (Under $\mathrm{CH}$ this fact follows from results of $[8$.) On the other hand there are models of ZFC with only nonselective $P$-points over $\omega$, models with a sole (necessarily selective) $P$-point (up to isomorphism), and also models without $P$-points (see [18]). The relationships between Hausdorff ultrafilters and other special classes $(P$ - and $Q$-points, arrow ultrafilters, etc.) will be investigated in a forthcoming paper.

Be as it may, so far as we do not abide ZFC as our foundational theory, we cannot prove that Hausdorff ultrafilters exist at all. In fact the forcing models recently defined by Bartoszynski and Shelah in [1] have no Hausdorff ultrafilters, even in the weaker sense that considers only $<\omega$-to- 1 functions in the condition $(\mathrm{H})$.

3.4. Some open questions. The results of the preceding sections suggest several questions. We itemize below a few of those that seem most interesting to us.

1. Is it consistent that a product of Hausdorff incompatible ultrafilters be nonHausdorff? and that $\mathcal{U} \otimes \mathcal{V}$ is Hausdorff whereas $\mathcal{V} \otimes \mathcal{U}$ is not?

2. Is the existence of Hausdorff ultrafilters derived from set-theoretic hypotheses weaker than those providing selective ultrafilters? (E.g. from $\mathfrak{x}=\mathfrak{c}$, for suitable cardinal invariant $\mathfrak{x}$ of the continuum?)

3. Is it consistent with ZFC that there are regular Hausdorff ultrafilters on sets of size greater than $\aleph_{1}$ ?

4. Assuming some large cardinal axiom, is it consistent with $\mathrm{ZFC}+\neg \mathrm{CH}$ that there are uniform Hausdorff ultrafilters on the continuum?

5 . What about nonnormal Hausdorff $\kappa$-ultrafilters?

\section{ACKNOWLEDGEMENTS}

The authors are grateful to Vieri Benci and Karel Hrbàcek for useful discussions, to Andreas Blass for several basic references, and to Tomek Bartoszynski for interesting suggestions and for providing a copy of [1].

\section{REFERENCES}

1. A. Bartoszynski, S. Shelah, There may be no Hausdorff ultrafilters, manuscript (2003, arXiv:math.LO/0311064).

2. J.E. Baumgartner, A.D. Taylor, Partition theorems and ultrafilters, Trans. Amer. Math. Soc. 241 (1978), 283-309. MR0491193 (58:10458)

3. V. Benci, M. Di Nasso, M. Forti, Hausdorff nonstandard extensions, Bol. Soc. Parana. Mat. (3) 20 (2002), 9-20. MR2010860 (2004h:54019)

4. A. Blass, The Rudin-Keisler ordering of P-points, Trans. Amer. Math. Soc. 179 (1973), 79-90. MR 0354350 (50:6830)

5. A. Blass, Combinatorial cardinal characteristics of the continuum, to appear in Handbook of Set Theory (M. Foreman, M. Magidor, A. Kanamori, eds.).

6. A. Blass, G. Moche, Finite preimages under the natural map from $\beta(\mathbb{N} \times \mathbb{N})$ to $\beta \mathbb{N} \times \beta \mathbb{N}$, Topology Proceedings (to appear).

7. C.C. Chang, H.J. Keisler, Model Theory (3rd edition), North-Holland, Amsterdam 1990. MR.1059055 (91c:03026)

8. M. Daguenet-Teissier, Ultrafiltres à la façon de Ramsey, Trans. Amer. Math. Soc. 250 (1979), 91-120. MR0530045 (81b:04004)

9. M. Di Nasso, M. Forti, Topological and nonstandard extensions, Monatsh. f. Math. 144 (2005), 89-112. MR2123958 (2005j:03050)

10. T. Jech, Set Theory (3rd edition), Springer, Berlin 2002.

11. A. Kanamori, Ultrafilters over a measurable cardinal, Ann. Math. Logic 11 (1977), 315-332. MR0491186 (58:10451) 
12. A. Kanamori, Finest partitions for ultrafilters, J. Symb. Logic 511 (1986), 327-356. MR0840409 (87f:04004)

13. A. Kanamori, A.D. Taylor, Separating ultrafilters on uncountable cardinals, Israel J. Math. 47 (1984), 131-138. MR0738164(85h:03058)

14. J. Ketonen, Ultrafilters over measurable cardinals, Fund. Math. 77 (1973), 257-269. MR0329897 (48:8237)

15. K. Kunen, Some applications of iterated ultrapowers in set theory, Ann. Math. Logic 1 (1970), 179-227. MR0277346 (43:3080)

16. A.H. Mekler, D.H. Pelletier, A.D. Taylor, A note on a lemma of Shelah concerning stationary sets, Proc. Amer. Math. Soc. 83 (1981), 764-768. MR0630051(82k:04007)

17. S. Ng, H. Render, The Puritz order and its relationship to the Rudin-Keisler order, in Reuniting the Antipodes - Constructive and Nonstandard Views of the Continuum (P. Schuster, U. Berger and H. Osswald, eds.), Synthèse Library 306, Kluwer A. P., New York 2001, 157166. MR1895391 (2003d:54045)

18. S. Shelah, Proper and Improper Forcing (2nd edition), Springer, Berlin 1998. MR 1623206 (98m:03002)

19. E.K. van Douwen, The integers and topology, in Handbook of Set Theoretic Topology (K. Kunen, J.E. Vaughan, eds.), North-Holland, Amsterdam 1984, 111-168. MR0776622 (87f:54008)

Dipartimento di Matematica "L. Tonelli", Universitì di Pisa, Italy

E-mail address: dinasso@dm.unipi.it

Dipartimento di Matematica Applicata "U. Dini", Università di Pisa, Italy

E-mail address: forti@dma.unipi.it 